\title{
Distribution of Different Forms of Potassium of Representative Soil Series of Sub-Montane Zone of Maharashtra, India
}

\author{
M.V.V.I. Annapurna*, B.S. Kadam and H.K. Surabhi \\ Department of Soil Science and Agricultural Chemistry, College of Agriculture, Kolhapur, \\ Mahatma Phule Krishi Vidyapeeth, Rahuri, Maharashtra, 413 722, India \\ *Corresponding author
}

\begin{tabular}{|c|c|}
\hline & A B S T R A C T \\
\hline Keywords & \multirow{9}{*}{$\begin{array}{l}\text { The representative soil series of order Entisols, Inceptisols and Vertisols collected } \\
\text { from agriculture college Kolhapur and different research stations of Sub-montane zone } \\
\text { of Maharashtra were assessed for different forms of potassium and its distribution. } \\
\text { The water soluble } \mathrm{K} \text { contributed very lowest fraction than the rest of all forms of } \\
\text { potassium (i.e. } 0.23 \% \text { of total K). From the mean values higher water soluble K was } \\
\text { noticed in Vertisols followed by Inceptisols and Entisols. Higher value of water } \\
\text { soluble } \mathrm{K} \text { was observed in surface layer and decreased with depth. Exchangeable K, } \\
\text { non-exchangeable K and lattice K contributed } 2.87,8.97 \text { and } 87.81 \text { per cent of total K, } \\
\text { respectively. From the mean values, highest exchangeable K, non-exchangeable K, } \\
\text { lattice K and total K were noticed in Vertisols followed by Inceptisols and Entisols. } \\
\text { There was no any specific trend noticed with respect to depth wise distribution of } \\
\text { different forms of potassium. }\end{array}$} \\
\hline Water soluble K, & \\
\hline $\begin{array}{l}\text { Exchangeable K, } \\
\text { Non-exchangeable }\end{array}$ & \\
\hline K, Lattice K, Total & \\
\hline $\begin{array}{l}\text { K and Sub- } \\
\text { montane zone. }\end{array}$ & \\
\hline Article Info & \\
\hline Accepted: & \\
\hline 23 June 2017 & \\
\hline $\begin{array}{l}\text { Available Online: } \\
10 \text { July } 2017\end{array}$ & \\
\hline
\end{tabular}

\section{Introduction}

Potassium is one of the three major plant nutrient elements. Its importance in Indian agriculture has increased with intensification of agriculture. Potassium is an essential nutrient element for all living organisms including plants and animals. It is a univalent cation found in the largest concentration in the plant cell sap and so it is called a "master cation". Potassium is ionic $\left(\mathrm{K}^{+}\right)$, free (not bound to any constituent) and mobile in plants. Potassium plays a vital roles in enzyme activation, water relations (osmotic regulation), energy relations, translocation of assimilates, photosynthesis, protein and starch synthesis (Mengel and Krikby, 1987). Over sixty enzymes require $\mathrm{K}$ for their activation.
In soils, potassium exists in different forms viz. water soluble, exchangeable, nonexchangeable and lattice potassium. The water soluble and exchangeable together constitutes the plant available potassium.

The information on vertical distribution of potassium in agricultural soils is important because it indicates the distribution of potassium with respect to depth of soils. It also indicate the depletion as well as accumulation pattern of potassium, if any within the profile. The present studies were, therefore, undertaken to evaluate the distribution of different forms of $\mathrm{K}$ for the 
representative soil series of Sub-montane zone of Maharashtra.

\section{Materials and Methods}

Horizon-wise twelve profile samples from representative soil series of order Entisols, Inceptisols and Vertisols from agriculture college, Kolhapur and different research stations of Sub-montane zone of Maharashtra were collected.

The collected soil samples were analysed for different forms of $\mathrm{K}$. Water soluble $\mathrm{K}$ was determined in a 1:5 soil: water extract (USSLS, 1954); exchangeable K by Knudsen et al., (1982); non-exchangeable $\mathrm{K}$ by boiling $1 \mathrm{~N} \mathrm{HNO}_{3}$ (Wood and De Turk, 1941); lattice $\mathrm{K}$ was calculated by subtracting $1 N \mathrm{HNO} 3$ extractable $\mathrm{K}$ from total $\mathrm{K}$. Total $\mathrm{K}$ was determined by extracting soil with $\mathrm{H}_{2} \mathrm{SO}_{4}$, $\mathrm{HClO}_{4}$ and $\mathrm{HF}$ mixture in platinum crucible at 220-225 ${ }^{\circ} \mathrm{C}$ (Jackson, 1973). Potassium estimation in the extracts was carried out with the help of a flame photometer.

\section{Results and Discussion}

The horizon wise distribution of different forms of $\mathrm{K}$ in different soil series of Entisols, Inceptisols and Vertisols presented in the tables 1,2 and 3 .

\section{Water soluble $\mathrm{K}$}

The average mean value of water soluble $\mathrm{K}$ in different pedons is $11.57 \mathrm{mg} \mathrm{kg}$. It contributed 0.23 per cent of total K. In general, most of the soil series showed comparatively higher water soluble $\mathrm{K}$ in surface horizon than sub-surface horizon.

This variation might be due to nature and intensities of cropping pattern, clay content, weathering stages of $\mathrm{K}$ bearing minerals and organic matter content in soil. Similar results were reported by Subba Rao et al., (1991) and Raskar and Pharande (1997).

\section{Exchangeable K}

The average mean value of exchangeable $\mathrm{K}$ in different soil series was $150.47 \mathrm{mg} \mathrm{kg}^{-1}$ and it contributed 2.87 per cent of total $\mathrm{K}$. The exchangeable $\mathrm{K}$ status in surface horizon was comparatively higher than sub surface horizons.

The higher exchangeable $\mathrm{K}$ status of surface layer could be due to application of $K$ fertilizers, crop residue, high organic carbon content and higher biological activities. These findings were similar with the results observed by Raskar and Pharande (1997) for black soils of Maharashtra.

\section{Non-exchangeable K}

The average mean value of non-exchangeable $\mathrm{K}$ in different soil series was $457.63 \mathrm{mg} \mathrm{kg}^{-1}$ and it contributed 8.97 per cent of total K. Most of the soil series showed comparatively low non-exchangeable $\mathrm{K}$ status in surface horizon than subsurface horizon.

As per the categorization proposed by Subba Rao et al., (1993) for non-exchangeable potassium reserve in all the soil series showed medium to high in non-exchangeable $\mathrm{K}$ status. The medium content of nonexchangeable $\mathrm{K}$-status might be due to low content of $\mathrm{K}$ bearing minerals such as muscovite, biotite and illite in clay fractions.

The higher status of non-exchangeable $\mathrm{K}$ in some soil series might be due to higher pedochemical weathering of $\mathrm{K}$ bearing minerals in soil and transformation into illite and vermiculite. The values of nonexchangeable $\mathrm{K}$ obtained were in agreement with those reported by Bhosale et al., (1992). 
Table.1 Forms of K of Entisol soil series

\begin{tabular}{|c|c|c|c|c|c|c|c|c|c|c|c|}
\hline \multirow{2}{*}{$\begin{array}{l}\text { Sr. } \\
\text { No. }\end{array}$} & \multirow[b]{2}{*}{ Horizon } & \multirow{2}{*}{$\begin{array}{c}\text { Depth } \\
(\mathrm{cm})\end{array}$} & \multicolumn{2}{|c|}{ Water soluble K } & \multicolumn{2}{|c|}{ Exchangeable K } & \multicolumn{2}{|c|}{ Non-Exchangeable K } & \multicolumn{2}{|c|}{ Lattice $\mathrm{K}$} & \multirow{2}{*}{$\begin{array}{c}\text { Total K } \\
\left(\mathrm{mg} \mathrm{kg}^{-1}\right)\end{array}$} \\
\hline & & & $\mathbf{m g ~ k g} \mathbf{~}^{-1}$ & $\begin{array}{c}\% \text { of } \\
\text { Total K }\end{array}$ & $\mathrm{mg} \mathrm{kg}^{-1}$ & $\begin{array}{c}\% \text { of Total } \\
\text { K }\end{array}$ & $\mathrm{mg} \mathrm{kg}^{-1}$ & $\begin{array}{c}\% \text { of Total } \\
\text { K }\end{array}$ & $\mathbf{m g ~ k g}^{-1}$ & $\begin{array}{c}\% \text { of Total } \\
\text { K }\end{array}$ & \\
\hline \multirow[t]{4}{*}{ I } & \multicolumn{11}{|c|}{ Ahmedpur series - A.R.S., Karad (Lithic ustorthents) } \\
\hline & $\mathrm{Ap}$ & $0-21$ & 11.03 & 0.24 & 113.40 & 2.46 & 396.36 & 8.61 & 4079.21 & 88.68 & 4600 \\
\hline & $\mathrm{A} 1$ & $21-45$ & 9.45 & 0.22 & 128.52 & 3.06 & 413.48 & 9.84 & 3648.55 & 86.87 & 4200 \\
\hline & Mean & & 10.24 & 0.23 & 120.96 & 2.76 & 404.92 & 9.23 & 3863.88 & 87.77 & 4400 \\
\hline \multirow[t]{7}{*}{ II } & \multicolumn{11}{|c|}{ Kurkum series - R.S. and J.R.S., Kolhapur (Lithic ustorthents) } \\
\hline & $\mathrm{Ap}$ & $0-22$ & 10.50 & 0.25 & 97.02 & 2.31 & 322.32 & 7.67 & 3770.16 & 89.76 & 4200 \\
\hline & A12 & $22-50$ & 8.93 & 0.23 & 124.74 & 3.28 & 406.56 & 10.70 & 3259.77 & 85.78 & 3800 \\
\hline & A13 & $50-80$ & 7.88 & 0.17 & 116.82 & 2.54 & 394.86 & 8.58 & 4080.44 & 88.7 & 4600 \\
\hline & A14 & 80-107 & 8.40 & 0.19 & 104.94 & 2.38 & 361.06 & 8.20 & 3925.60 & 89.22 & 4400 \\
\hline & A15 & $>107$ & 8.93 & 0.20 & 100.98 & 2.29 & 346.40 & 7.87 & 3943.69 & 89.63 & 4400 \\
\hline & Mean & & 8.93 & 0.21 & 108.90 & 2.56 & 366.24 & 8.60 & 3795.93 & 88.62 & 4300 \\
\hline \multirow[t]{5}{*}{ III } & \multicolumn{11}{|c|}{ Sathesai series - N.A.R.P.(S.Z), Shendapark (Lithic ustorthents) } \\
\hline & Ap & $0-25$ & 8.40 & 0.26 & 90.34 & 2.82 & 287.40 & 8.98 & 2813.86 & 87.93 & 3200 \\
\hline & A1 & $25-50$ & 6.30 & 0.22 & 103.76 & 3.58 & 333.24 & 11.49 & 2456.70 & 84.71 & 2900 \\
\hline & A2 & $>50$ & 7.35 & 0.19 & 97.28 & 2.56 & 306.68 & 8.07 & 3388.69 & 89.18 & 3800 \\
\hline & Mean & & 7.35 & 0.22 & 97.13 & 2.99 & 309.11 & 9.51 & 2886.42 & 87.27 & 3300 \\
\hline \multirow[t]{4}{*}{ IV } & \multicolumn{11}{|c|}{ Kurkum series - Agriculture college, Kolhapur (Lithic ustorthents) } \\
\hline & Ap & $0-18$ & 8.93 & 0.23 & 104.94 & 2.76 & 321.79 & 8.47 & 3364.34 & 88.53 & 3800 \\
\hline & A1 & $18-30$ & 7.88 & 0.18 & 118.42 & 2.75 & 360.54 & 8.38 & 3813.16 & 88.68 & 4300 \\
\hline & Mean & & 8.41 & 0.21 & 111.68 & 2.75 & 341.17 & 8.42 & 3588.75 & 88.60 & 4100 \\
\hline \multirow[t]{5}{*}{$\mathbf{V}$} & \multicolumn{11}{|c|}{ Kurkum series - A.R.S., Vadgaon-Maval (Lithic ustorthents) } \\
\hline & Ap & $0-20$ & 8.93 & 0.25 & 105.84 & 2.94 & 318.78 & 8.85 & 3166.45 & 87.96 & 3600 \\
\hline & A1 & $20-45$ & 7.35 & 0.17 & 115.96 & 2.63 & 382.46 & 8.69 & 3894.23 & 88.50 & 4400 \\
\hline & A2 & $>45$ & 8.40 & 0.20 & 94.50 & 2.25 & 343.82 & 8.19 & 3753.28 & 89.36 & 4200 \\
\hline & Mean & & 8.22 & 0.20 & 105.43 & 2.61 & 348.35 & 8.58 & 3604.65 & 88.61 & 4100 \\
\hline \multicolumn{2}{|c|}{ Avg. Mean } & & 8.63 & 0.21 & 108.82 & 2.71 & 353.96 & 8.85 & 3547.93 & 88.12 & 4000 \\
\hline \multicolumn{2}{|c|}{ Range } & & $6.30-11.03$ & $0.17-0.26$ & $\begin{array}{c}90.34- \\
128.52 \\
\end{array}$ & $2.25-3.58$ & $\begin{array}{c}287.40- \\
413.48 \\
\end{array}$ & $\begin{array}{l}7.67- \\
11.49 \\
\end{array}$ & $\begin{array}{c}2456.7- \\
4080.44 \\
\end{array}$ & $\begin{array}{c}84.71- \\
89.76 \\
\end{array}$ & $\begin{array}{c}2900- \\
4600 \\
\end{array}$ \\
\hline
\end{tabular}


Table.2 Forms of K of Inceptisol soil series

\begin{tabular}{|c|c|c|c|c|c|c|c|c|c|c|c|}
\hline \multirow{2}{*}{$\begin{array}{l}\text { Sr. } \\
\text { No. }\end{array}$} & \multirow[b]{2}{*}{ Horizon } & \multirow{2}{*}{$\begin{array}{c}\text { Depth } \\
(\mathbf{c m})\end{array}$} & \multicolumn{2}{|c|}{ Water soluble $\mathbf{K}$} & \multicolumn{2}{|c|}{ Exchangeable $\mathrm{K}$} & \multicolumn{2}{|c|}{ Non-Exchangeable $\mathbf{K}$} & \multicolumn{2}{|c|}{ Lattice $\mathbf{K}$} & \multirow{2}{*}{$\begin{array}{c}\text { Total K } \\
\left(\mathrm{mg} \mathrm{kg}^{-1}\right)\end{array}$} \\
\hline & & & $\mathrm{mg} \mathrm{kg}^{-1}$ & $\begin{array}{c}\% \text { of Total } \\
\text { K }\end{array}$ & $\mathrm{mg} \mathrm{kg}^{-1}$ & $\begin{array}{c}\text { \% of Total } \\
\text { K }\end{array}$ & $\mathrm{mg} \mathrm{kg}^{-1}$ & $\begin{array}{c}\% \text { of Total } \\
\text { K }\end{array}$ & $\mathrm{mg} \mathrm{kg}^{-1}$ & $\begin{array}{c}\text { \% of Total } \\
\text { K }\end{array}$ & \\
\hline \multirow[t]{7}{*}{$\mathbf{I}$} & \multicolumn{11}{|c|}{ Kankauli - A.R.S., Radhanagari (Typic haplusterts) } \\
\hline & Ap & $0-20$ & 12.85 & 0.30 & 139.86 & 3.33 & 335.58 & 7.99 & 3711.71 & 88.37 & 4200 \\
\hline & $\mathrm{B} 21$ & $20-45$ & 8.93 & 0.19 & 113.40 & 2.46 & 360.56 & 7.84 & 4117.11 & 89.50 & 4600 \\
\hline & B22 & $45-75$ & 10.50 & 0.20 & 117.18 & 2.30 & 425.11 & 8.33 & 4547.21 & 89.16 & 5100 \\
\hline & B23 & $75-90$ & 8.40 & 0.18 & 124.74 & 2.65 & 382.41 & 8.14 & 4184.45 & 89.03 & 4700 \\
\hline & B24 & $90-115$ & 7.35 & 0.17 & 103.62 & 2.41 & 374.63 & 8.71 & 3814.40 & 88.71 & 4300 \\
\hline & Mean & & 9.61 & 0.21 & 119.76 & 2.63 & 375.66 & 8.20 & 4074.98 & 88.95 & 4600 \\
\hline \multirow[t]{7}{*}{ II } & \multicolumn{11}{|c|}{ Bamburdi series - Agriculture college, Kolhapur (Typic ustochrepts) } \\
\hline & Ap & $0-15$ & 14.70 & 0.29 & 207.64 & 4.07 & 430.99 & 8.24 & 4446.67 & 87.19 & 5100 \\
\hline & B21 & $15-30$ & 9.45 & 0.17 & 174.32 & 3.17 & 407.89 & 8.33 & 4908.34 & 89.24 & 5500 \\
\hline & B22 & $30-46$ & 12.60 & 0.21 & 192.40 & 3.15 & 520.45 & 8.32 & 5374.55 & 88.11 & 6100 \\
\hline & B23 & $46-64$ & 10.50 & 0.18 & 186.12 & 3.26 & 496.20 & 8.36 & 5007.18 & 87.84 & 5700 \\
\hline & B24 & $>64$ & 8.40 & 0.16 & 176.22 & 3.45 & 447.09 & 8.29 & 4468.29 & 87.61 & 5100 \\
\hline & Mean & & 11.13 & 0.20 & 187.34 & 3.42 & 460.52 & 8.31 & 4841.01 & 88.00 & 5500 \\
\hline III & \multicolumn{11}{|c|}{ Bamburdi series -A.R.S., Vadgaon-Maval (Typic ustochrepts) } \\
\hline & Ap & $0-20$ & 13.65 & 0.26 & 144.54 & 2.73 & 415.80 & 8.32 & 4726.01 & 89.17 & 5300 \\
\hline & B21 & $20-45$ & 9.45 & 0.20 & 106.94 & 2.23 & 396.36 & 8.32 & 4287.25 & 89.32 & 4800 \\
\hline & B22 & $45-75$ & 10.50 & 0.19 & 153.76 & 2.79 & 436.78 & 8.32 & 4898.96 & 89.07 & 5500 \\
\hline & B23 & $75-90$ & 8.93 & 0.16 & 101.68 & 1.78 & 511.07 & 8.31 & 5078.32 & 89.09 & 5700 \\
\hline & B24 & 90-105 & 7.88 & 0.15 & 146.52 & 2.76 & 473.58 & 8.32 & 4672.02 & 88.15 & 5300 \\
\hline & Mean & & 10.08 & 0.19 & 130.69 & 2.46 & 446.72 & 8.32 & 4732.51 & 88.96 & 5300 \\
\hline \multicolumn{2}{|c|}{ Avg. Mean } & & 10.27 & 0.20 & 145.93 & 2.84 & 427.63 & 8.28 & 4549.50 & 88.64 & 5100 \\
\hline \multicolumn{2}{|c|}{ Range } & & $\begin{array}{l}7.35- \\
14.70 \\
\end{array}$ & $0.15-0.30$ & $\begin{array}{c}101.68- \\
207.64\end{array}$ & $1.78-4.07$ & $\begin{array}{c}335.58- \\
520.45\end{array}$ & $7.84-8.71$ & $\begin{array}{c}3711.71- \\
5374.55 \\
\end{array}$ & $\begin{array}{c}87.19- \\
89.50\end{array}$ & $4200-6100$ \\
\hline
\end{tabular}


Table.3 Forms of K of Vertisol soil series

\begin{tabular}{|c|c|c|c|c|c|c|c|c|c|c|c|}
\hline \multirow[b]{2}{*}{$\begin{array}{l}\text { Sr. } \\
\text { No. }\end{array}$} & \multirow[b]{2}{*}{ Horizon } & \multirow{2}{*}{$\begin{array}{l}\text { Depth } \\
\text { (cm) }\end{array}$} & \multicolumn{2}{|c|}{ Water soluble K } & \multicolumn{2}{|c|}{ Exchangeable K } & \multicolumn{2}{|c|}{ Non-Exchangeable $\mathbf{K}$} & \multicolumn{2}{|c|}{ Lattice K } & \multirow[b]{2}{*}{$\begin{array}{c}\text { Total K } \\
\left(\mathrm{mg} \mathrm{kg}^{-1}\right)\end{array}$} \\
\hline & & & $\mathrm{mg} \mathrm{kg}^{-1}$ & $\begin{array}{c}\% \text { of Total } \\
\text { K }\end{array}$ & $\mathrm{mg} \mathrm{kg}^{-1}$ & $\begin{array}{c}\% \text { of Total } \\
\mathrm{K}\end{array}$ & $\mathrm{mg} \mathrm{kg}^{-1}$ & $\begin{array}{c}\% \text { of Total } \\
\mathrm{K}\end{array}$ & $\mathrm{mg} \mathrm{kg}^{-1}$ & $\begin{array}{c}\% \text { of Total } \\
K\end{array}$ & \\
\hline $\mathbf{I}$ & \multicolumn{11}{|c|}{ Koregaon series - A.R.S., Karad (Typic haplusterts) } \\
\hline & Ap & $0-24$ & 19.70 & 0.35 & 241.92 & 4.32 & 629.02 & 11.23 & 4709.36 & 84.10 & 5600 \\
\hline & A12 & $24-54$ & 17.88 & 0.34 & 219.24 & 4.14 & 577.54 & 10.90 & 4485.34 & 84.63 & 5300 \\
\hline & A13 & $54-85$ & 16.80 & 0.28 & 230.58 & 3.91 & 680.09 & 11.53 & 4972.53 & 84.28 & 5900 \\
\hline & A14 & $85-108$ & 18.30 & 0.29 & 223.02 & 3.48 & 718.83 & 11.23 & 5439.85 & 85.00 & 6400 \\
\hline & A15 & $>108$ & 15.85 & 0.22 & 215.46 & 2.95 & 646.52 & 8.86 & 6422.17 & 87.97 & 7300 \\
\hline & Mean & & 17.71 & 0.30 & 226.04 & 3.76 & 650.40 & 10.75 & 5205.85 & 85.20 & 6100 \\
\hline II & \multicolumn{11}{|c|}{ Shiware series - R.S. and J.R.S., Kolhapur (Typic haplusterts) } \\
\hline & Ap & $0-24$ & 19.70 & 0.34 & 219.24 & 3.85 & 586.94 & 10.30 & 4877.02 & 85.56 & 5700 \\
\hline & $\mathrm{B} 21$ & $24-54$ & 17.88 & 0.29 & 200.34 & 3.23 & 531.02 & 8.56 & 5454.99 & 87.98 & 6200 \\
\hline & $\mathrm{B} 22$ & $54-85$ & 16.80 & 0.28 & 192.78 & 3.27 & 558.76 & 9.47 & 5133.76 & 87.01 & 5900 \\
\hline & B23 & $85-108$ & 18.30 & 0.28 & 204.12 & 3.19 & 643.96 & 10.06 & 5536.07 & 86.50 & 6400 \\
\hline & $\mathrm{AC}$ & $>108$ & 15.85 & 0.24 & 196.20 & 2.93 & 580.58 & 8.66 & 5910.62 & 88.22 & 6700 \\
\hline & Mean & & 14.72 & 0.29 & 202.54 & 3.29 & 580.25 & 9.41 & 5382.49 & 87.05 & 6200 \\
\hline III & \multicolumn{11}{|c|}{ Koregaon series - A.R.S., Gadhinglaj (Typic haplusterts) } \\
\hline & Ap & $0-23$ & 18.30 & 0.33 & 238.14 & 4.33 & 591.33 & 10.75 & 4652.23 & 84.59 & 5500 \\
\hline & B1 & $23-55$ & 17.24 & 0.28 & 204.12 & 3.35 & 566.90 & 9.29 & 5311.74 & 87.08 & 6100 \\
\hline & Bss1 & $55-85$ & 16.80 & 0.29 & 223.02 & 3.91 & 665.77 & 11.68 & 4794.41 & 84.11 & 5700 \\
\hline & Bss2 & $85-105$ & 17.88 & 0.30 & 234.36 & 3.97 & 686.32 & 11.63 & 4961.44 & 84.09 & 5900 \\
\hline & $\mathrm{AC}$ & $>105$ & 15.85 & 0.26 & 215.46 & 3.53 & 643.47 & 10.55 & 5225.22 & 85.66 & 6100 \\
\hline & Mean & & 17.21 & 0.29 & 223.02 & 3.82 & 630.76 & 10.78 & 4989.01 & 85.11 & 5900 \\
\hline IV & \multicolumn{11}{|c|}{ Donoli series - Agriculture college, Kolhapur (Udic haplusterts) } \\
\hline & Ap & $0-20$ & 15.85 & 0.26 & 154.98 & 2.54 & 464.38 & 7.61 & 5464.79 & 89.59 & 6100 \\
\hline & A12 & $20-55$ & 13.65 & 0.25 & 142.30 & 2.59 & 454.55 & 8.26 & 4889.5 & 88.90 & 5500 \\
\hline & A13 & $55-90$ & 12.60 & 0.20 & 136.08 & 2.16 & 511.07 & 8.11 & 5640.25 & 89.53 & 6300 \\
\hline & A14 & $90-105$ & 14.70 & 0.23 & 124.74 & 1.92 & 558.08 & 8.58 & 5802.48 & 89.27 & 6500 \\
\hline & A15 & $>105$ & 11.03 & 0.17 & 117.18 & 1.86 & 531.02 & 8.43 & 5640.77 & 89.54 & 6300 \\
\hline & Mean & & 13.57 & 0.22 & 135.06 & 2.21 & 503.82 & 8.20 & 5487.56 & 89.37 & 6100 \\
\hline \multicolumn{2}{|c|}{ Avg. Mean } & & 15.80 & 0.28 & 196.66 & 3.27 & 591.31 & 9.79 & 5266.23 & 86.68 & 6100 \\
\hline \multicolumn{2}{|c|}{ Range } & & $\begin{array}{c}11.03- \\
19.70 \\
\end{array}$ & $\begin{array}{l}0.17- \\
0.35 \\
\end{array}$ & $\begin{array}{c}117.18- \\
241.92 \\
\end{array}$ & $\begin{array}{l}1.86- \\
4.33\end{array}$ & $\begin{array}{c}454.55- \\
718.83\end{array}$ & $\begin{array}{l}7.61- \\
11.68 \\
\end{array}$ & $\begin{array}{c}4485.34- \\
6422.17 \\
\end{array}$ & $\begin{array}{c}84.09- \\
89.59 \\
\end{array}$ & $5300-7300$ \\
\hline \multicolumn{3}{|c|}{ Overall series Mean } & 11.57 & 0.23 & 150.47 & 2.87 & 457.63 & 8.97 & 4454.55 & 87.81 & 5100 \\
\hline \multicolumn{3}{|c|}{ Overall series Range } & $6.30-19.70$ & $\begin{array}{l}0.15- \\
0.35 \\
\end{array}$ & $90.34-241.92$ & $\begin{array}{l}1.78- \\
4.33 \\
\end{array}$ & $\begin{array}{c}287.40- \\
718.83\end{array}$ & $\begin{array}{l}7.61- \\
11.68 \\
\end{array}$ & $\begin{array}{c}2456.70- \\
6422.17 \\
\end{array}$ & $\begin{array}{c}84.68- \\
89.76\end{array}$ & $2900-7300$ \\
\hline
\end{tabular}




\section{Lattice K}

The average mean value of lattice $\mathrm{K}$ in different soil series was $4454.55 \mathrm{mg} \mathrm{kg}^{-1}$ and it contributed 87.81 per cent of total $\mathrm{K}$. Thus these results suggest that the majority of total $\mathrm{K}$ was located in clay mineral lattices. The lattice $\mathrm{K}$ values of different soil series were low in surface horizon than sub surface horizon. No definite was observed regarding the depth wise distribution of lattice $\mathrm{K}$. The variation in lattice $\mathrm{K}$ might be due to degree of weathering of $\mathrm{K}$ bearing minerals in soils.

\section{Total K}

The average mean value of total $\mathrm{K}$ in different soil series was $5100 \mathrm{mg} \mathrm{kg}^{-1}$. Surface horizon of most of the soil series showed lower total K than sub surface horizon indicating pedochemical weathering of $\mathrm{K}$ bearing in surface horizon than subsurface horizons. On the basis of total K status proposed by Subba Rao et al., (1993), from the mean values, it was observed that the total $\mathrm{K}$ content of Vertisols and Inceptisols soil order were medium. Whereas, the soil series of Entisols soil order was low to medium in total $\mathrm{K}$ status. The values of total $\mathrm{K}$ were in agreement with the values reported by Kadrekar and Kibe (1972), Murthy (1988) and Sharma and Dubey (1988). In conclusion, the present study revealed considerable variation in the distribution of different forms of $\mathrm{K}$ in the horizons of the different soil samples collected from the representative soil series of sub-montane zone of Maharashtra. The study pointed out the need of integrated use of organic manures and $\mathrm{K}$ fertilizers for ensuring steady supply of $\mathrm{K}$ to crops to sustain production in the long run.

\section{References}

\section{How to cite this article:}

Annapurna, M.V.V.I., B.S. Kadam and Surabhi, H.K. 2017. Distribution of Different Forms of Potassium of Representative Soil Series of Sub-Montane Zone of Maharashtra. Int.J.Curr.Microbiol.App.Sci. 6(7): 26482653. doi: https://doi.org/10.20546/ijcmas.2017.607.313 